\title{
Predicting the Microstructural Evolution of Electron Beam Melting of Alloy 718 with Phase-Field Modeling
}

\author{
CHAMARA KUMARA, DUNYONG DENG, FABIAN HANNING, \\ MORTEN RAANES, JOHAN MOVERARE, and PER NYLÉN
}

\begin{abstract}
Electron beam melting (EBM) is a powder bed additive manufacturing process where a powder material is melted selectively in a layer-by-layer approach using an electron beam. EBM has some unique features during the manufacture of components with high-performance superalloys that are commonly used in gas turbines such as Alloy 718. EBM has a high deposition rate due to its high beam energy and speed, comparatively low residual stresses, and limited problems with oxidation. However, due to the layer-by-layer melting approach and high powder bed temperature, the as-built EBM Alloy 718 exhibits a microstructural gradient starting from the top of the sample. In this study, we conducted modeling to obtain a deeper understanding of microstructural development during EBM and the homogenization that occurs during manufacturing with Alloy 718. A multicomponent phase-field modeling approach was combined with transformation kinetic modeling to predict the microstructural gradient and the results were compared with experimental observations. In particular, we investigated the segregation of elements during solidification and the subsequent "in situ" homogenization heat treatment at the elevated powder bed temperature. The predicted elemental composition was then used for thermodynamic modeling to predict the changes in the continuous cooling transformation and time-temperature transformation diagrams for Alloy 718, which helped to explain the observed phase evolution within the microstructure. The results indicate that the proposed approach can be employed as a valuable tool for understanding processes and for process development, including post-heat treatments.
\end{abstract}

https://doi.org/10.1007/s11661-019-05163-7

(C) The Author(s) 2019

\section{INTRODUCTION}

RECENTLY, powder bed additive manufacturing (AM) has attracted great interest from the manufacturing industries and research community because of its capacity to produce near net shape structures with complex geometries, which cannot be manufactured

CHAMARA KUMARA and PER NYLÉN are with the Division of Subtractive and Additive Manufacturing Processes, Department of Engineering Science, University West, 46186 Trollhättan, Sweden. Contact e-mail: chamara.kumara@hv.se DUNYONG DENG is with the Division of Engineering Materials, Department of Management and Engineering, Linköping University, 58183 Linköping, Sweden. FABIAN HANNING is wiht the Department of Industrial and Materials Science, Chalmers University of Technology, 41296 Göteborg, Sweden. MORTEN RAANES is with the Department of Materials Science and Engineering, IMA, NTNU, Alfred Getz vei 2,7491 Trondheim, Norway. JOHAN MOVERARE is with the Division of Subtractive and Additive Manufacturing Processes, Department of Engineering Science, University West and also with the Division of Engineering Materials, Department of Management and Engineering, Linköping University.

Manuscript submitted November 21, 2018.

Article published online February 27, 2019 with traditional methods. Among the powder bed manufacturing processes, electron beam melting (EBM) process has attracted more attention because if its relatively higher productivity due to the high beam speed and high beam power density. In addition, the EBM operation occurs at a high temperature in a vacuum environment, which creates less residual stress and less oxidation in the component obtained. ${ }^{[1]}$ These features are beneficial for the manufacture of the critical components used in aerospace applications and gas turbine engines.

Nickel-based superalloys are among the most important alloys used in aerospace applications and gas turbine engines because of their high-temperature strength, high resistance to creep deformation, and corrosion resistance. ${ }^{[2,3]}$ Among these superalloys, Alloy 718 is one of the most widely used nickel-iron-based superalloys and it is suitable for AM processes because of its good weldability due to the sluggish precipitation of the main strengthening phase $\gamma^{\prime \prime} .{ }^{[4]}$ The microstructure of Alloy 718 is dominated by an austenitic $\gamma$ fcc matrix. Precipitates such as Laves, $\gamma^{\prime} / \gamma^{\prime \prime}$, and $\delta$ phases, and various metallic carbides and nitrides can be found within the matrix. The formation of the Laves phase is 
usually observed in the interdendritic region due to the segregation of the elements. The complete microstructure, including the phases present as well as their distribution, morphology, and orientation, is mainly related to the primary manufacturing technology employed and the subsequent post-processing conditions. Heat treatments are commonly used to tailor the microstructure of Alloy 718 to obtain the desired properties required for the application.

Due to the inherent features of the layer-by-layer manufacturing approach, the microstructure of Alloy 718 after the EBM process exhibits a gradient along the build direction. ${ }^{[5,6]}$ During the melting process, the powder material is melted and it then solidifies, thereby leading to the formation of different phases, as mentioned earlier. As the subsequent layers are built, the solidified structure gradually undergoes "in situ" heat treatment due to the elevated powder bed temperature $\left(>1000{ }^{\circ} \mathrm{C}\right.$ for Alloy 718 ) in the EBM process. The time that a specific layer undergoes this "in situ" heat treatment changes according to the height of the object under construction, which creates a gradient in the microstructure from the top to the bottom of the sample.

In this study, we modeled the microstructure using the multiphase-field method and the transformation kinetics were determined to understand the formation of the microstructural gradient in Alloy 718 samples produced using EBM. First, the solidified microstructure was modeled and the model was then used to simulate the "in situ" heat treatment in order to observe the changes in the alloy composition and any subsequent phase changes. The results were compared with experimental observations.

\section{EXPERIMENTAL}

A plasma atomized powder (nominal size ranges from 25 to $106 \mu \mathrm{m}$ ) supplied by Arcam AB was used to manufacture the Alloy 718 samples in this study. The chemical composition of the powder is shown in Table I.

Table I. Nominal Chemical Composition of the Raw Powder and the Nominal Composition Used for the Phase-Field Simulation

\begin{tabular}{lcc}
\hline Element (Weight Percent) & Measured & Simulation \\
\hline $\mathrm{Ni}$ & bal. & bal. \\
$\mathrm{Cr}$ & 19.1 & 19.1 \\
$\mathrm{Fe}$ & 18.5 & 18.5 \\
$\mathrm{Nb}$ & 5.04 & 5.04 \\
$\mathrm{Mo}$ & 2.95 & 2.95 \\
$\mathrm{Co}$ & 0.07 & - \\
$\mathrm{Ti}$ & 0.91 & 0.91 \\
$\mathrm{Al}$ & 0.58 & 0.58 \\
$\mathrm{Mn}$ & 0.05 & - \\
$\mathrm{Si}$ & 0.13 & - \\
$\mathrm{Cu}$ & 0.1 & - \\
$\mathrm{C}$ & 0.035 & - \\
$\mathrm{N}$ & 0.0128 & - \\
\hline
\end{tabular}

An Arcam A2X EBM system was used to manufacture the samples with the standard settings for Alloy 718 (listed in Table II). The manufacturing process started after the powder bed was pre-heated to about $1020{ }^{\circ} \mathrm{C}$ (measured under the base plate) and this temperature was maintained throughout the whole process. Each deposition cycle comprised (1) pre-heating the current powder layer, (2) contour melting the frame for the build, (3) hatch melting the interior of the build and rotating about $65^{\circ}$ from the previous scanning vector, (4) post-heating the current layer, and (5) lowering down the powder bed and raking new powder to form a uniform layer measuring $75 \mu \mathrm{m}$ for the next cycle. In each batch, 16 identical-sized blocks were fabricated and the dimension of each block was approximately $35 \mathrm{~mm}$ (length) $\times 10 \mathrm{~mm}$ (width) $\times 33 \mathrm{~mm}$ (height)

Cross-sections parallel to the build direction were examined at different heights from the top surface in order to characterize the microstructural gradient. Samples were mounted, mechanically ground successively from 500 grit to 4000 grit, and polished with a diamond suspension from 3 to $1 / 4 \mu \mathrm{m}$, and then finally with OP-U colloidal silica suspension. A Hitachi SU70 FEG scanning electron microscope (SEM) that operated at an accelerating voltage of $20 \mathrm{kV}$, which was equipped with an energy dispersive X-ray spectroscopy system, was employed to determine the microstructural features and chemical compositions. In order to calculate the volume fraction of the Laves phase, SEM images were converted into binary images using the Image J program, before distinguishing the contrast between the matrix and Laves $+\mathrm{NbC}$ phases. Electron probe microscopic analysis was performed using a JEOL JXA-8500F system with samples that were cut normal to the build direction.

\section{MODELING}

\section{A. MICRESS and the Governing Equation}

The phase-field method was employed to model the evolution of the microstructure. This method has been used widely during the last two decades to simulate the microstructural evolution of materials. ${ }^{[7,8]}$ The advantage of the phase-field method is that there is no need to track the interface, unlike the classical sharp interface modeling methods. An order parameter is introduced that varies smoothly between two phases, and thus the

Table II. Main Parameters of the Arcam Standard Parameters for Alloy 718 (Theam Name-"'Inconel 718 Melt $75 \mu \mathrm{m} \mathrm{V3')}$

\begin{tabular}{ll}
\hline Parameter & \multicolumn{1}{c}{ Value } \\
\hline Hatch-current max $(\mathrm{mA})$ & 18 \\
Hatch-scan speed $(\mathrm{m} / \mathrm{s})$ & automatic (scan function 63$)$ \\
Hatch-line offset $(\mathrm{mm})$ & 0.125 \\
Pre-heating temperature $\left({ }^{\circ} \mathrm{C}\right)$ & 1025 \\
Layer thickness $(\mu \mathrm{m})$ & 75 \\
Electron beam power $(\mathrm{W})$ & 3000 \\
\hline
\end{tabular}


interface is part of the solution in the phase-field method.

Our simulations were performed using the commercially available phase-field modeling software MICRESS (version 6.400, Access e.V., Aachen, Germany). MICRESS is based on the multiphase-field approach. ${ }^{[9,10]}$ The multiphase-field theory describes the evolution of multiple phase-field parameters $\phi_{\alpha=1,2, \ldots, v}=(\vec{x}, t)$ (with the constraint $\sum_{\alpha=1}^{v} \phi_{\alpha}=1$ ) in space and time, which represent the spatial distribution of multiple phases with different thermodynamic properties and/or multiple grains with different orientations. The phase-field parameter, $\phi_{\alpha}$ takes a value of 1 if phase $\alpha$ is present locally and a value of 0 if the phase is not present locally. At the interface of the phase $\alpha, \phi_{\alpha}$ will vary smoothly from 0 to 1 over the interface thickness $(\eta)$. The time evolution of $\phi_{\alpha}$ is calculated using the free energy functional, $F$, which integrates the density functional, $f$, over the domain $\Omega$.

$$
F\left(\left\{\phi_{\alpha}\right\},\left\{\vec{C}_{\alpha}\right\}\right)=\int_{\Omega} f\left(\left\{\phi_{\alpha}\right\},\left\{\vec{C}_{\alpha}\right\}\right),
$$

where the brackets, \{\} , represent all phases of $\alpha$, and not an individual $\alpha$. The density functional, $f$, depends on the interface energy density, $f^{\text {int }}$, and chemical free energy, $f^{\text {chem }}$, and thus it can be written as follows:

$$
\begin{gathered}
f=f^{\text {int }}\left\{\left\{\phi_{\alpha}\right\}+f^{\text {chem }}\left\{\left\{\phi_{\alpha}\right\},\left\{\vec{C}_{\alpha}\right\}\right\},\right. \\
f=\sum_{\alpha=1}^{v} \sum_{\beta \neq \alpha}^{v} \frac{4 \sigma_{\alpha \beta}^{0} a_{\alpha \beta}^{\sigma}}{v \eta}\left(-\frac{\eta^{2}}{\pi^{2}} \nabla \phi_{\alpha} \nabla \phi_{\beta}+\phi_{\alpha} \phi_{\beta}\right) \\
+\sum_{\alpha=1}^{v} \phi_{\alpha} f_{\alpha}\left(\vec{C}_{\alpha}\right),
\end{gathered}
$$

where $\sigma_{\alpha \beta}^{0}$ represents the interfacial energy of the interface between $\alpha$ and $\beta$. v is the total number of local coexisting phases. The term $a_{\alpha \beta}^{\sigma}$ represents the anisotropy function for the interfacial stiffness. ${ }^{[1]}$ In $2 \mathrm{D}$, for cubic crystal systems, this function takes the form $a_{\alpha \beta}^{\sigma}=1-\delta_{\sigma} \cos (4 \theta){ }^{[12]}$

The multiphase-field equation defining the time evolution of $\phi_{\alpha}=(\vec{x}, t)$ in multiple phase transformations is derived by minimizing the total free energy, $F$, according to a relaxation principle.

$$
\dot{\phi}_{\alpha}=\sum_{\beta \neq \alpha}^{v} M_{\alpha \beta} a_{\alpha \beta}^{M}\left(\frac{\delta F}{\delta \phi_{\beta}}-\frac{\delta F}{\delta \phi_{\alpha}}\right)
$$

Here $M_{\alpha \beta}$ is the mobility of the $\alpha$ and $\beta$ interface. The term $a_{\alpha \beta}^{M}$ represents the anisotropy function for the interfacial mobility. ${ }^{[11]}$ In $2 \mathrm{D}$, for cubic crystal systems, this function takes the form $a_{\alpha \beta}^{M}=1+\delta_{M} \cos (4 \theta){ }^{[12]}$

The general version of the evolution equation including the anisotropy can be written as follows.

$$
\begin{gathered}
\dot{\phi}_{\alpha}=\sum_{\beta \neq \alpha}^{v} M_{\alpha \beta} a_{\alpha \beta}^{M}\left[b_{\alpha \beta} \Delta G_{\alpha \beta}-\sigma_{\alpha \beta}^{0} a_{\alpha \beta}^{\sigma} K_{\alpha \beta}^{a}+\sum_{\gamma \neq \beta \neq \alpha}^{n} J_{\alpha \beta \gamma}\right] \\
b_{\alpha \beta}=\frac{\pi}{\eta}\left(\phi_{\alpha}+\phi_{\beta}\right)\left(\sqrt{\phi_{\alpha} \phi_{\beta}}\right) \\
K_{\alpha \beta}^{a}=\frac{2}{v}\left\{\frac{\pi^{2}}{2 \eta^{2}}\left(\phi_{\beta}-\phi_{\alpha}\right)+\frac{1}{2}\left(\nabla^{2} \phi_{\beta}-\nabla^{2} \phi_{\alpha}\right)+\frac{1}{a_{\alpha \beta}^{\sigma}} \sum_{i=1}^{3} \nabla_{i}\right. \\
\times\left[\left(\frac{\partial a_{\alpha \beta}^{\sigma}}{\partial \nabla_{i} \phi_{\beta}}-\frac{\partial a_{\alpha \beta}^{\sigma}}{\partial \nabla_{i} \phi_{\alpha}}\right)\left(\frac{\pi^{2}}{2 \eta^{2}}\left(\phi_{\alpha} \phi_{\beta}\right)-\frac{1}{2}\left(\nabla \phi_{\alpha} \nabla \phi_{\beta}\right)\right)\right] \\
\left.-\frac{1}{a_{\alpha \beta}^{\sigma}} \nabla a_{\alpha \beta}^{\sigma}\left(\nabla \phi_{\beta}-\nabla \phi_{\alpha}\right)\right\}
\end{gathered}
$$

$$
\begin{aligned}
J_{\alpha \beta \gamma} & =\frac{2}{v}\left\{\frac{1}{2}\left(\sigma_{\beta \gamma}^{0} a_{\beta \gamma}^{\sigma}-\sigma_{\alpha \gamma}^{0} a_{\alpha \gamma}^{\sigma}\right)\left(\frac{\pi^{2}}{\eta^{2}} \phi_{\gamma}+\nabla^{2} \phi_{\gamma}\right)\right. \\
+ & \sigma_{\alpha \gamma}^{0} \sum_{i=1}^{3} \nabla_{i}\left[\left(\frac{\partial a_{\alpha \gamma}^{\sigma}}{\partial \nabla_{i} \phi_{\alpha}}\right)\left(\frac{\pi^{2}}{2 \eta^{2}}\left(\phi_{\alpha} \phi_{\gamma}\right)-\frac{1}{2}\left(\nabla \phi_{\alpha} \nabla \phi_{\gamma}\right)\right)\right] \\
& -\sigma_{\beta \gamma}^{0} \sum_{i=1}^{3} \nabla_{i}\left[\left(\frac{\partial a_{\beta \gamma}^{\sigma}}{\partial \nabla_{i} \phi_{\beta}}\right)\left(\frac{\pi^{2}}{2 \eta^{2}}\left(\phi_{\beta} \phi_{\gamma}\right)-\frac{1}{2}\left(\nabla \phi_{\beta} \nabla \phi_{\gamma}\right)\right)\right] \\
+ & \left.\frac{1}{2}\left(\sigma_{\beta \gamma}^{0} \nabla a_{\beta \gamma}^{\sigma}-\sigma_{\alpha \gamma}^{0} \nabla a_{\alpha \gamma}^{\sigma}\right) \nabla \phi_{\gamma}\right\},
\end{aligned}
$$

where $K_{\alpha \beta}^{a}$ is related to the local curvature of the interface and $J_{\alpha \beta \gamma}$ relates to the third-order junction forces.

However, more simplified version of the $J_{\alpha \beta \gamma}$ term is implemented in MCRESS neglecting the higher order terms as follows.

$$
J_{\alpha \beta \gamma}=\frac{2}{v}\left\{\frac{1}{2}\left(\sigma_{\beta \gamma}^{0} a_{\beta \gamma}^{\sigma}-\sigma_{\alpha \gamma}^{0} a_{\alpha \gamma}^{\sigma}\right)\left(\frac{\pi^{2}}{\eta^{2}} \phi_{\gamma}+\nabla^{2} \phi_{\gamma}\right)\right\} .
$$

The interface motion depends on the curvature contribution, $\left(\sigma_{\alpha \beta} K_{\alpha \beta}\right)$, but also on the thermodynamic driving force, $\Delta G_{\alpha \beta}(\vec{C}, T)$. This driving force depends on the temperature, $T$, and the local multicomponent composition, $\vec{C}$, which couples the phase-field equation to the multiphase diffusion equations:

$$
\begin{gathered}
\dot{\vec{C}}=\nabla \sum_{\alpha=1}^{v} \phi_{\alpha} \vec{D}_{\alpha} \nabla \vec{C}_{\alpha} \\
\vec{C}=\sum_{\alpha=1}^{v} \phi_{\alpha} \vec{C}_{\alpha},
\end{gathered}
$$


where $\vec{D}_{\alpha}$ represents the multicomponent diffusion coefficient matrix for the phase $\alpha . \Delta G_{\alpha \beta}(\vec{C}, T)$ and $\vec{D}_{\alpha}$ are calculated by direct coupling to the thermodynamic (TCNI8) and mobility (MOBNI4) databases via the TQ-interface in Thermo-Calc Software. ${ }^{[13]}$ The driving force, $\Delta G_{\alpha \beta}(\vec{C}, T)$, is calculated based on the quasiequilibrium approach with the combination of mass balance condition. For detail information, reader is advised to refer. ${ }^{[10,11]}$

\section{B. Model Setup in MICRESS and Assumptions}

Two-dimensional (2D) multiphase-field simulations were conducted in the present study. The 2D domain selected was normal to the build direction of the EBM sample. Therefore, the domain had an isothermal cross section and it was normal to the primary dendrite growth direction. The unit cell approach proposed by Warnken et al. ${ }^{[14]}$ was employed, where the edge length of the unit cell was given by primary dendrite arm spacing (PDAS) and the unit cell contained one representative dendrite. Therefore, we considered a unit cell size of $6 \mu \mathrm{m} \times 6 \mu \mathrm{m}$ (the PDAS was measured experimentally based on SEM images) with a grid spacing of $0.025 \mu \mathrm{m}$ for the EBM solidification simulation. Alloy 718 was modeled as a seven-component system with the composition shown in Table I. This simplifying assumption reduced the computational effort required to calculate the thermodynamic and mobility data. Both of these types of data were dynamically extracted from the TCNI8 and MOBNI4 databases by Thermo-Calc. In addition, we considered the full multicomponent diffusion matrix based on the local composition values.

The simulation started from a complete liquid state with the composition in Table I. The $\gamma$ phase nucleation seed was placed at the center of the domain. Measuring the cooling rate of the EBM process is rather difficult due to the inherent nature of the process. Therefore, a value of $2000 \mathrm{~K} / \mathrm{s}$ was assumed for the simulation, which is in the range of the cooling rate values reported for EBM Alloy $718 .^{[6]}$ Periodic boundary conditions were assigned at the boundaries of the simulated domain.

During the solidification process, various phases such as TiN, MC, and Laves phases begin to precipitate from the liquid. ${ }^{[4]}$ However, in the present study, we only considered the formation of the Laves phase. This simplifying assumption reduced the complexity of the model and the computational effort required. However, TiN and MC could not be modeled because the simplified alloy system did not contain $\mathrm{N}$ and $\mathrm{C}$. This simplification can be justified as follows.

I. The $\mathrm{N}$ and $\mathrm{C}$ proportions (wt pct) in the alloy were comparatively low compared with those of the other major elements.

II. The observed volume fractions of nitrides and carbides were very low in the microstructure. Therefore, the consumption of $\mathrm{Ti}$ and $\mathrm{Nb}$ during the formation of nitrides and carbides was not significant and it did not significantly influence the formation of the other phases.

III. The abundances of carbides and nitrides did not vary significantly throughout the build height of the sample.

In addition, the formation of the strengthening phases, $\gamma^{\prime} / \gamma^{\prime \prime}$, was not modeled. A very small grid resolution (typically in the rage of $1 \mathrm{~nm}$ ) is required in order to capture the formation of these nano-scale precipitates, thereby demanding greater computational effort.

The modeled Laves phase was allowed to nucleate at the liquid $-\gamma$ interface. In order to simulate the eutectic formation of Laves $+\gamma$, the nucleation site for eutectic $\gamma$ was allowed to form at the liquid-Laves interface. For both types of nucleation, a critical undercooling value of $2 \mathrm{~K}$ was set. To simplify the simulation, only the liquid $/ \gamma$ interface was modeled as an anisotropic interface with cubic crystal anisotropy. ${ }^{[15]}$ The parameters used in the simulations are summarized in Table III.

According to the thermocouple measurements (see Figure 1) obtained from the bottom of the base plate in the EBM system, the temperature of the base plate was around $1020{ }^{\circ} \mathrm{C}$ throughout the build time. We assumed that the entire build volume was in isothermal equilibrium with the thermocouple at this temperature during the process. This "in situ" heat treatment changed the solidified microstructure. Therefore, the heat treatment simulation was performed at $1020^{\circ} \mathrm{C}$ in order to observe its effects on the solidified microstructure. The microstructure obtained from the solidification simulation (solidified microstructure) was used as the initial microstructure for the in situ heat treatment simulation.

The homogenization behavior observed in the EBM solidified microstructure in the simulations and experiments was more rapid than the homogenization behavior observed in the cast Alloy 718. Therefore, for comparative purposes, the hypothetical cast microstructure formation and subsequent homogenization heat treatment were modeled in a similar manner. A PDAS of $100 \mu \mathrm{m}$ was selected for the cast solidification microstructure simulations. ${ }^{[16]}$ Therefore, the domain size was $100 \mu \mathrm{m} \times 100 \mu \mathrm{m}$ with a grid resolution of $0.5 \mu \mathrm{m}$. A cooling rate of $1 \mathrm{~K} / \mathrm{s}$ was employed. ${ }^{[16]}$ For the homogenization heat treatment, a temperature value of $1100{ }^{\circ} \mathrm{C}$ was used according to AMS5383E.$^{[17]}$

\section{Calculation of Continuous Cooling Transformation (CCT) and Time-Temperature Transformation (TTT) Diagrams Using JMatPro}

The $\gamma^{\prime} / \gamma^{\prime \prime}$ and $\delta$ phase precipitation processes were not modeled in the multiphase-field simulations. However, in order to observe their kinetic behavior during precipitation due to element segregation, CCT diagrams were generated using the JMatPro (ver10.2) material modeling software package. ${ }^{[18]}$ The nominal alloy composition and the segregated compositions predicted by the multiphase-field simulations were utilized in these simulations. 
Table III. Summery of the Model Parameters

\begin{tabular}{|c|c|c|}
\hline & EBM & As cast \\
\hline Domain size & $6 \mu \mathrm{m} \times 6 \mu \mathrm{m}$ & $100 \mu \mathrm{m} \times 100 \mu \mathrm{m}$ \\
\hline Grid resolution $(\Delta x)$ & $0.025 \mu \mathrm{m}$ & $0.5 \mu \mathrm{m}$ \\
\hline Interface thickness $(\eta)$ & $3 \cdot \Delta \mathrm{x}$ & $2.5 \cdot \Delta x$ \\
\hline Cooling Rate $(\mathrm{K} / \mathrm{s})$ & 2000 & 1 \\
\hline Initial undercooling for $\gamma^{* \dagger}(\mathrm{K})$ & 11 & 6 \\
\hline Interface energy liquid $/ \gamma\left(\mathrm{J} / \mathrm{cm}^{2}\right)$ & $1.2 \mathrm{E}-05^{[8]}$ & \\
\hline Anisotropic interfacial stiffness coefficient $\left(\boldsymbol{\delta}_{\boldsymbol{\sigma}}\right)^{*}$-liquid $/ \gamma$ & 0.2 & \\
\hline Anisotropic interfacial mobility coefficient $\left(\left(\boldsymbol{\delta}_{M}\right)^{*}\right.$-liquid $/ \gamma$ & 0.2 & \\
\hline Assumed interface energy liquid/laves $\left(\mathrm{J} / \mathrm{cm}^{2}\right)$ & $6 \mathrm{E}-06$ & \\
\hline Assumed interface energy $\gamma /$ laves $\left(\mathrm{J} / \mathrm{cm}^{2}\right)$ & $5 \mathrm{E}-06$ & \\
\hline
\end{tabular}

*Values were selected based on trial and error approach to get the desired dendrite morphology.

$\dagger$ The two different initial undercooling values are due to the two different initial nucleation size (due to the different resolution of the models) for the $\gamma$ phase.

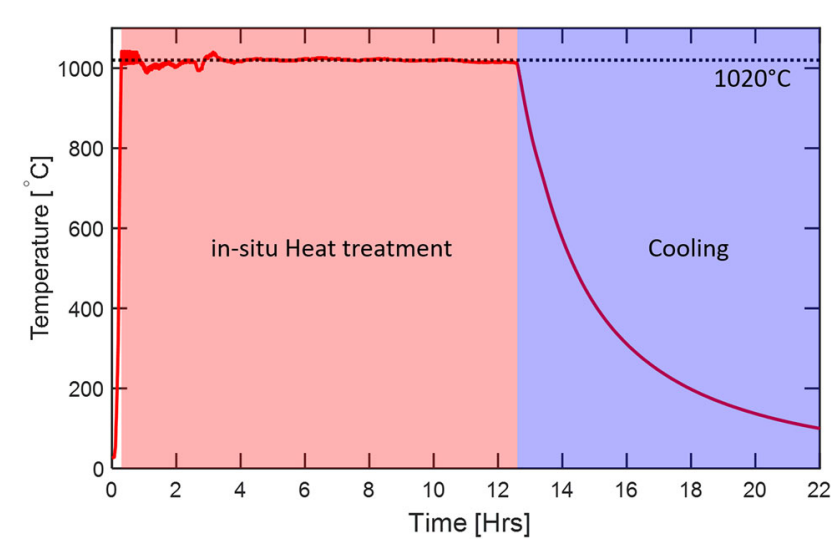

Fig. 1-Thermocouple measurement from the bottom of the build plate.

\section{RESULTS AND DISCUSSION}

\section{A. Experimental Results: "In Situ” Homogenization and Phase Formation}

In the following, we present the experimental results obtained from the microstructural observations that were most relevant for the modeling and validation studies. Detailed information regarding the microstructural characterization process and the results obtained were published previously. ${ }^{[19]}$

Experimental examinations of the microstructure of the sample clearly indicated the presence of a microstructure gradient along the build direction as well as from the dendrite core to the interdendritic region. This gradient along the build direction was visible when observing the bright particles in the interdendritic regions of the microstructure, as shown in Figure 2. These particles were confirmed as the Laves phase and $\mathrm{NbC} /(\mathrm{Nb}, \mathrm{Ti})(\mathrm{C}, \mathrm{N})$ according to transmission electron microscopy (TEM) analysis. The area fractions of these phases were measured by image analysis in order to determine the evolution of the gradients of these phases. The measured Laves $+\mathrm{NbC} /(\mathrm{Nb}, \mathrm{Ti})(\mathrm{C}, \mathrm{N})$ volume fractions are shown in Figure 3. In the area close to the top surface of the sample, a low volume fraction of Laves $+\mathrm{NbC} /(\mathrm{Nb}, \mathrm{Ti})(\mathrm{C}, \mathrm{N})$ was observed.

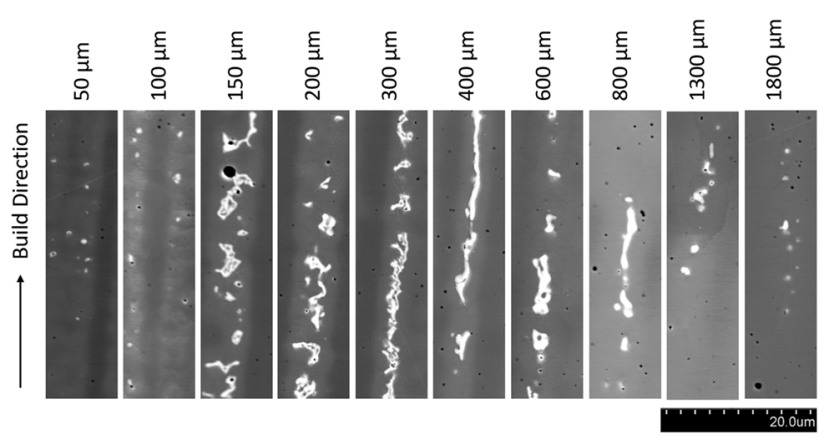

Fig. 2-Laves $+\mathrm{NbC} /(\mathrm{Nb}, \mathrm{Ti})(\mathrm{C}, \mathrm{N})$ phases morphology at different distance from the top surface.

However, the peak volume fraction was found at a depth of around $150 \mu \mathrm{m}$. Kirka et al. ${ }^{[6]}$ showed that melting current layer of the powder material in the EBM process will lead to re-melting of the top two layers below the current layer that is added. Therefore, the $225 \mu \mathrm{m}$ layer from the top of the sample can be considered as the "solidified" region without further re-melting. In the "solidified" region, the amount of Laves $+\mathrm{NbC} /(\mathrm{Nb}, \mathrm{Ti})(\mathrm{C}, \mathrm{N})$ phases decreased when moving closer to the top surface, which could be attributed to the change in the solidification velocity of the melt pool. Raghavan et al. ${ }^{[20]}$ showed that the initial solidification velocity is relatively lower during the solidification of the melt pool in the EBM process. When the solidification velocity is low and it is lower that the element diffusion velocity, the elements will have sufficient time to partition and segregate into the interdendritic region. However, the solidification velocity was shown to increase towards the end of the solidification of the melt pool. As the solidification velocity increases, more elements are increasingly trapped inside the dendrite, ${ }^{[21]}$ which results in less element segregation in the interdendritic region, thereby reducing the formation of Laves $+\mathrm{NbC} /(\mathrm{Nb}, \mathrm{Ti})(\mathrm{C}, \mathrm{N})$. It should be noted that this phenomenon was not modeled in the present study.

According to the temperature measurements shown in Figure 1, we expected that the powder bed temperature remained above $1020{ }^{\circ} \mathrm{C}$ throughout the building of the 

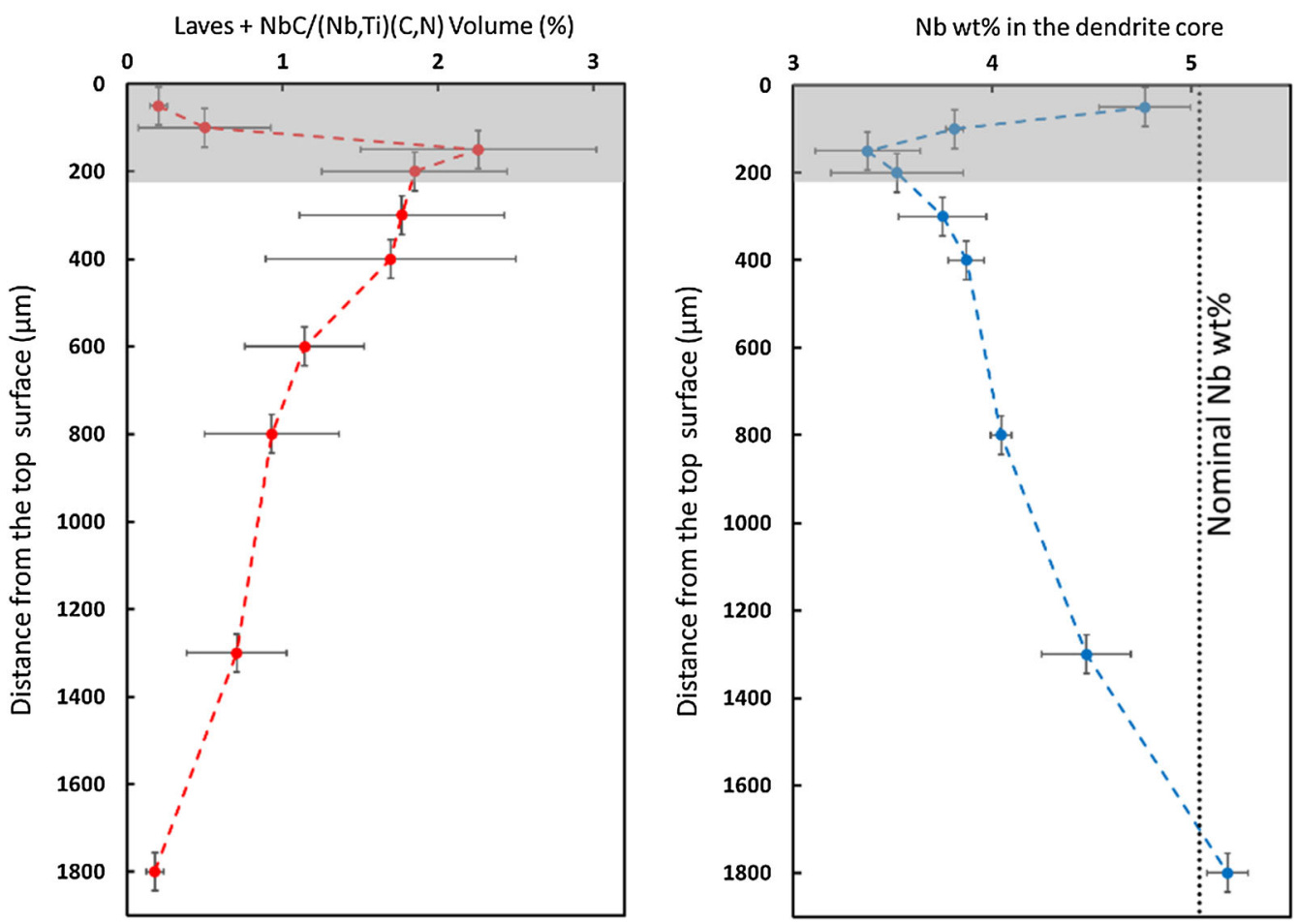

Fig. 3-Measured Laves $+\mathrm{NbC} /(\mathrm{Nb}, \mathrm{Ti})(\mathrm{C}, \mathrm{N})$ volume fraction and $\mathrm{Nb}$ wt pct in the dendrite core from top surface of the sample. Shaded areas represent the possible last-solidified region without subjecting to "in situ" heat treatment.

samples. Therefore, this elevated temperature acted as an "in situ" heat treatment and changed the "solidified" microstructure. The effect of this "in situ" heat treatment on the "solidified" microstructure was evident when moving away from the peak location for the Laves $+\mathrm{NbC} /(\mathrm{Nb}, \mathrm{Ti})(\mathrm{C}, \mathrm{N})$ phase, as shown in Figure 3. The volume fraction of the Laves $+\mathrm{NbC} /$ $(\mathrm{Nb}, \mathrm{Ti})(\mathrm{C}, \mathrm{N})$ phase started to decrease gradually as the distance increased, which can be attributed directly to the dissolution of the Laves phase during the "in situ" heat treatment. Due to their high stability, $\mathrm{NbC} /$ $(\mathrm{Nb}, \mathrm{Ti})(\mathrm{C}, \mathrm{N})$ were not affected by the "in situ" heat treatment, and thus their volume fractions were expected to remain unchanged with the build height. The Laves phase was no longer visible at a depth of $\sim 1800 \mu \mathrm{m}$ from the top surface and it was expected to be fully dissolved. This distance of $1800 \mu \mathrm{m}$ is roughly around the 30th layer counting from the top of the build sample. Considering the total build time and the total number of layers, we estimated that the 30th layer was exposed to "in situ" heat treatment at a time of roughly 40 minutes.

$\mathrm{Nb}$ is considered to be one of the most important alloying elements in Alloy 718. ${ }^{[22,23]}$ The formation of phases such as $\gamma^{\prime} / \gamma^{\prime \prime}$, Laves, and $\delta$ is directly related to the level of $\mathrm{Nb}$ in the microstructure. ${ }^{[22]} \mathrm{Nb}$ is also the most severely segregated element in the microstructure of Alloy 718, and thus it is relatively easy to measure its segregation. Figure 3 shows the variation in the proportion of $\mathrm{Nb}(\mathrm{Nb}$ wt pct) at the center of the dendrite core as a function of the distance from the top surface of the sample. The changes in $\mathrm{Nb}$ wt pct exhibited the opposite relationship to the variations in the Laves + $\mathrm{NbC} /(\mathrm{Nb}, \mathrm{Ti})(\mathrm{C}, \mathrm{N})$ volume fractions, thereby indicating that the $\mathrm{Nb}$ trapped inside the Laves phase in the "solidified" microstructure was released and it diffused back into the dendrite core as a consequence of the "in situ" heat treatment. At a distance of $\sim 1800 \mu \mathrm{m}$ from the top surface, the $\mathrm{Nb}$ wt pct in the dendrite core was similar to the nominal composition of the powder material used in this study, which indicates that the "solidified" microstructure tended to homogenize during the 40-min "in situ" heat treatment.

\section{B. Phase-Field Solidification Simulation Results of EBM alloy 718 and Cast Alloy 718}

During the solidification of Alloy 718, elements such as $\mathrm{Nb}, \mathrm{Mo}$, and $\mathrm{Ti}$ will segregate into the interdendritic region due to the low solubility of these elements in the $\gamma$-matrix. ${ }^{[22]}$ This elemental segregation leads to the formation of phases such as Laves, $\delta, \mathrm{NbC}$, and TiN. In addition, the depletion of these elements in the $\gamma$-matrix will affect the precipitation kinetics for the strengthening phases (which we illustrate later using CCT diagrams generated by JMatPro). Figure 4 shows the distribution maps obtained for $\mathrm{Nb}, \mathrm{Fe}$, and $\mathrm{Ti}$ based on the solidification simulation for EBM Alloy 718, which demonstrates that $\mathrm{Nb}$ and $\mathrm{Ti}$ were depleted inside the dendrite but enriched in the interdendritic region, whereas $\mathrm{Fe}$ exhibited the opposite variation. This discrepancy was due to the different partition coefficients of $\mathrm{Nb}, \mathrm{Ti}$, and $\mathrm{Fe}$ in the alloy system. The segregation of elements during solidification modified 

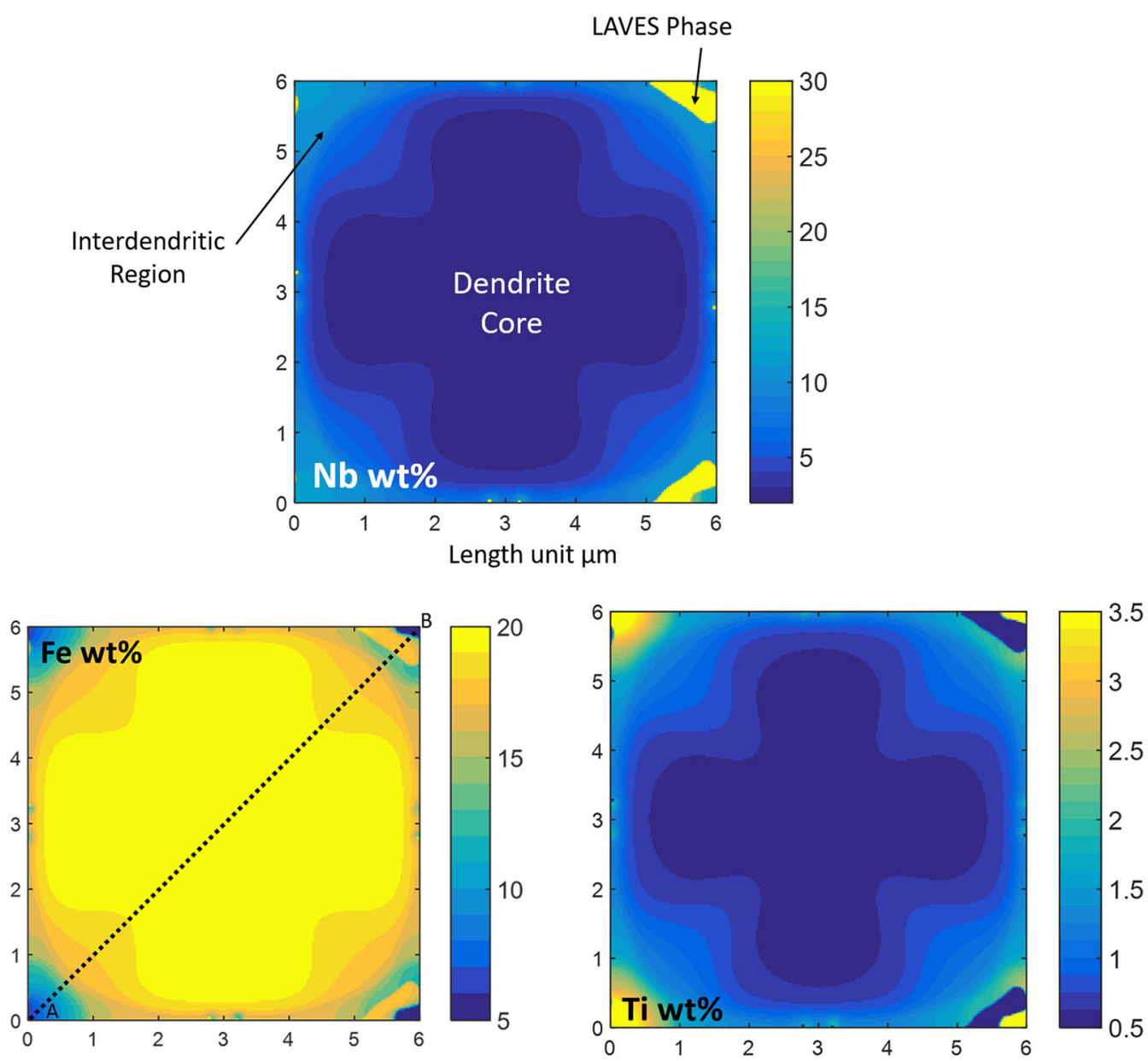

Fig. $4-\mathrm{Nb}, \mathrm{Fe}$, and $\mathrm{Ti}$, distribution maps at the end of the solidification of EBM Alloy 718.

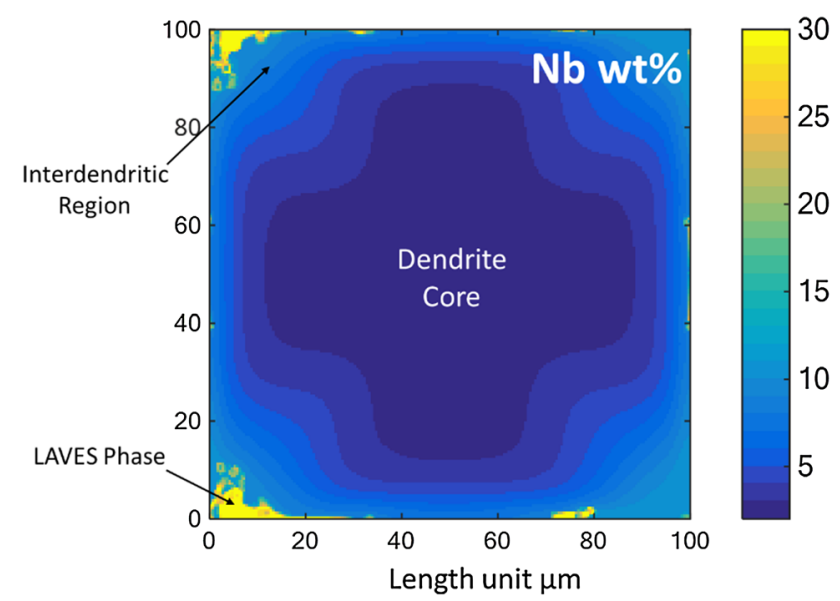

Fig. 5- $\mathrm{Nb}$ distribution maps at the end of the solidification of cast Alloy 718 simulation. Line AB was used to do the virtual EDX on the modeled microstructure.

the local thermodynamics and created the necessary driving force to form the Laves phase in the interdendritic region. Figure 5 shows the distribution map obtained for $\mathrm{Nb}$ at the end of the cast simulation, where the observed segregation behavior of the elements was similar to the EBM microstructure. The size of the Laves phase particles in the cast microstructure was larger than that in the EBM microstructure, which was related to the larger length solidification scale in the cast microstructure.

Table IV shows the compositions measured at the dendrite core and the Laves phase in both the phase-field model and the actual sample's microstructure. In the Laves phase, the amounts of $\mathrm{Nb}$ and $\mathrm{Mo}$ obtained by phase-field modeling differed considerably compared with the values measured in the composition of the EBM sample. These high $\mathrm{Nb}$ and low Mo values could be explained by errors in the TCNI8 database. A simple Scheil simulation was performed using ThermoCalc (using TCNI8 and MOBNI4 databases) to check the $\mathrm{Nb}$ and Mo contents of the Laves phase from the start of its formation. In Scheil simulation, it also predicted around $41 \mathrm{wt}$ pet $\mathrm{Nb}$ and $0.7 \mathrm{wt}$ pet Mo. According to the Thermo-Calc company, no parameters in the TCNI8 database have been assessed for the $\mathrm{Cr}-\mathrm{Nb}-\mathrm{Mo}$ system, which could have led to the high and low solubilities for $\mathrm{Nb}$ and $\mathrm{Mo}$ in the Laves phase, respectively. 
Table IV. Composition Measured in the Dendrite Core and Laves Phase Both from Phase-Field Model and Real Sample

\begin{tabular}{|c|c|c|c|c|c|c|c|c|}
\hline & & & $\mathrm{Al}$ & $\mathrm{Ti}$ & $\mathrm{Cr}$ & $\mathrm{Nb}$ & $\mathrm{Fe}$ & Mo \\
\hline \multirow{3}{*}{ Laves } & Model & & 0.16 & 0.32 & 15.32 & 40.85 & 17.72 & 0.88 \\
\hline & EPMA & Average & 0.20 & 0.86 & 14.33 & 28.52 & 13.36 & 6.87 \\
\hline & & Standard deviation & 0.06 & 0.05 & 0.92 & 1.37 & 0.24 & 0.35 \\
\hline \multirow{3}{*}{ Dendrite core } & Model & & 0.56 & 0.57 & 19.71 & 2.41 & 19.91 & 2.49 \\
\hline & EPMA & Average & 0.57 & 0.77 & 20.03 & 3.38 & 19.97 & 2.57 \\
\hline & & Standard deviation & 0.04 & 0.07 & 0.19 & 0.13 & 0.18 & 0.12 \\
\hline
\end{tabular}

\section{Homogenization Behavior of EBM Alloy 718 and Cast Alloy 718}

The homogenization heat treatments for Alloy 718 cast products are usually performed at a high temperature $\left(>1090{ }^{\circ} \mathrm{C}\right)$ for a sufficient time ( $>1$ hours) until the Laves phase dissolve. ${ }^{[2]}$ The Laves phase contains a high amount of $\mathrm{Nb}$, so dissolution of the Laves phase is important for redistributing the trapped $\mathrm{Nb}$, which is needed to form the strengthening phases. Nonetheless, even if the Laves dissolves, obtaining a homogeneous distribution of elements in the microstructure is not economically viable. ${ }^{[22]}$ However, as mentioned above, the observed homogenization of the elements in the microstructure of the EBM Alloy 718 samples occurred rather quickly ( 40 minutes) during the "in situ" heat treatment in the build process.

Figure 6 shows the elemental distributions of $\mathrm{Nb}, \mathrm{Fe}$, and $\mathrm{Ti}$ along the line $\mathrm{AB}$ (the line $\mathrm{AB}$ is shown in Figure 4) in the EBM Alloy 718 at the end of the solidification simulation ("as built") and during the "in situ" heat treatment simulation. The segregated elements in the as-built condition tended to homogenize after 40 minutes during the "in situ" heat treatment at around $1020^{\circ} \mathrm{C}$. Both $\mathrm{Nb}$ and $\mathrm{Ti}$ exhibited very low segregation after 40 minutes, whereas some segregation of Fe was still observed. This segregation is expected to be reduced by further "in situ" heat treatment and the microstructure is expected to reach its nominal composition.

However, the heat treatment simulation of the cast microstructure did not indicate the same homogenization compared with the EBM microstructure, as shown in Figure 7. Some of the Laves phase still remained at the end of the heat treatment simulation for the cast microstructure. By contrast, complete dissolution of the Laves phase was achieved in the heat treatment simulation of the EBM microstructure, which could have been related to the smaller size of the Laves phase particles in the EBM sample compared with the cast Alloy 718. Smaller particles will dissolve in a shorter time than larger particles. Another reason for the relatively rapid homogenization in the EBM microstructure is the smaller PDAS because the microstructure obtained in the EBM process will have a relatively smaller ( one order of magnitude smaller) PDAS compared with cast products. This difference will lead to segregation at a finer scale and a smaller diffusion length for the elements. As a consequence, EBM microstructures will tend to homogenize more rapidly compared with cast microstructures. It has been has
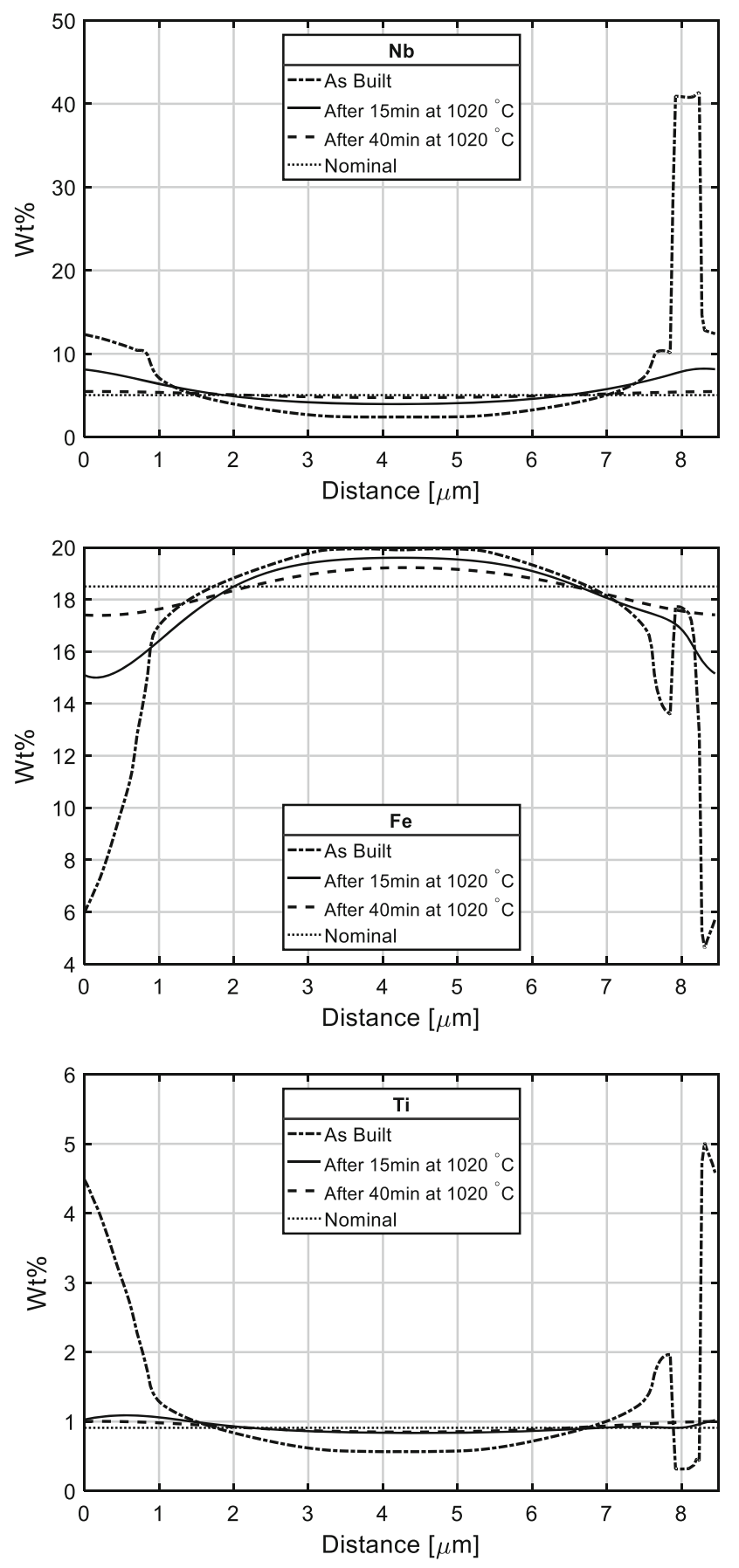

Fig. 6- Nb, Fe, and Ti variation along the "AB" virtual EDX line in the EBM microstructure-simulated domain. 


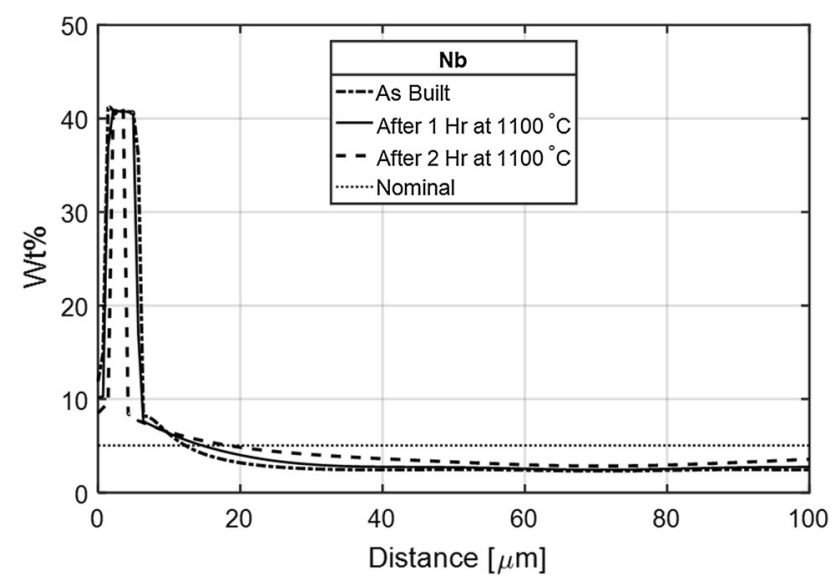

Fig. 7- Nb variation along the "AB" virtual EDX line in the cast microstructure-simulated domain.

shown that other AM processes such as laser metal directed energy deposition and selective laser melting of Alloy 718 resulted PDAS values with a similar order of magnitude to EBM, ${ }^{[8,24]}$ which indicates that the segregated microstructures produced in these processes can be homogenized rather rapidly compared with cast products. This difference could facilitate the design of new heat treatment protocols for AM microstructures.

\section{Change in Precipitation Kinetics of Phases in the Microstructure}

The precipitation kinetics of an alloy depend on the local composition levels. The local composition of the microstructure in Alloy 718 differs from its nominal composition value because of the segregation of the elements during solidification. A segregated microstructure behaves in a different manner compared with a microstructure in the nominal composition of the same alloy, ${ }^{[25]}$ which is illustrated based on the CCT diagrams obtained (as explained in Section III-C) for Alloy 718 in the following.

As mentioned above, during the building of the sample, the temperature of the build volume was around $1020{ }^{\circ} \mathrm{C}$ or above. This temperature is greater than the solvus temperature for $\gamma^{\prime} / \gamma^{\prime \prime}$ and around the solvus temperature for $\delta,{ }^{[26,27]}$ which implies that the formation of these phases could have occurred during the cooling stage of the build process. After the last layer was built, helium gas was blown in to cool the build chamber, as shown by the thermocouple measurements in Figure 1. During the cooling process, the temperature dropped through the precipitation temperature ranges for $\gamma^{\prime} / \gamma^{\prime \prime}$ and $\delta$.

In the "solidified" microstructure of the EBM sample, relatively higher amounts of $\gamma^{\prime} / \gamma^{\prime \prime}$ and $\delta$ were observed close to the Laves phase. The density of these precipitates decayed when moving away from the Laves phase, as shown in Figure 8. Similar observations were reported previously for Alloy 718 built by direct laser additive manufacturing. ${ }^{[28]}$ We produced CCT diagrams using JMatPro for compositions close to the Laves phase and in the dendrite core based on phase-field

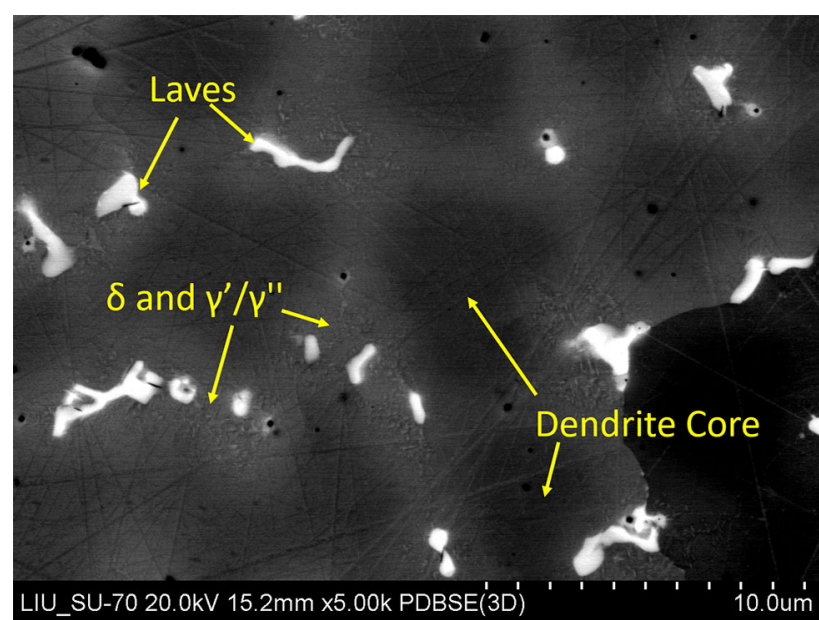

Fig. 8- SEM that shows the Laves phase and precipitation around it. (Image has been taken from a section Normal to the Build direction). It should be noted that $\gamma^{\prime} / \gamma^{\prime \prime}$ precipitates close to the Laves phase have been mainly observed through TEM analysis work. Ref. [19] for more information about the TEM work.

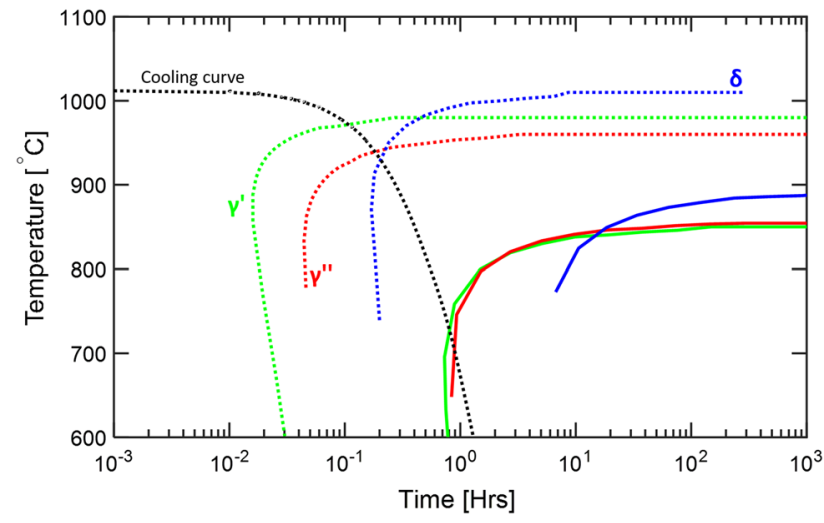

Fig. 9-CCT diagram created using JMatPro. Dotted line represents the 0.5 pct transformation close to Laves phase and solid line represent $0.5 \mathrm{pct}$ transformation in the dendrite core. The cooling curve has been created from the thermocouple measurement in the cooling stage in Fig. 1.

simulations in order to explain the observed gradient in the precipitates. As shown in Figure 9, the precipitation kinetics were altered due to the change in the local composition, where $\gamma^{\prime} / \gamma^{\prime \prime}$ and $\delta$ precipitated much earlier close to the Laves phase (more than an order of magnitude in time) compared with the core of the dendrite. The accelerated kinetics with the combination of change in local equilibrium conditions due to the local change in the composition, led to a higher density of the $\gamma^{\prime} / \gamma^{\prime \prime}$ and $\delta$ phases close to the Laves phase. Due to the lower density of $\gamma^{\prime} / \gamma^{\prime \prime}$ in the dendrite core of the "solidified" microstructure, the hardness measured in the dendrite core was expected to be low compared with that in the interdendritic region.

Figure 10 shows the CCT curves obtained based on the nominal composition of the alloy and the dendrite core composition of the "solidified microstructure. As the "in situ" heat treatment progressed, the elemental segregation in the "solidified" microstructure became 


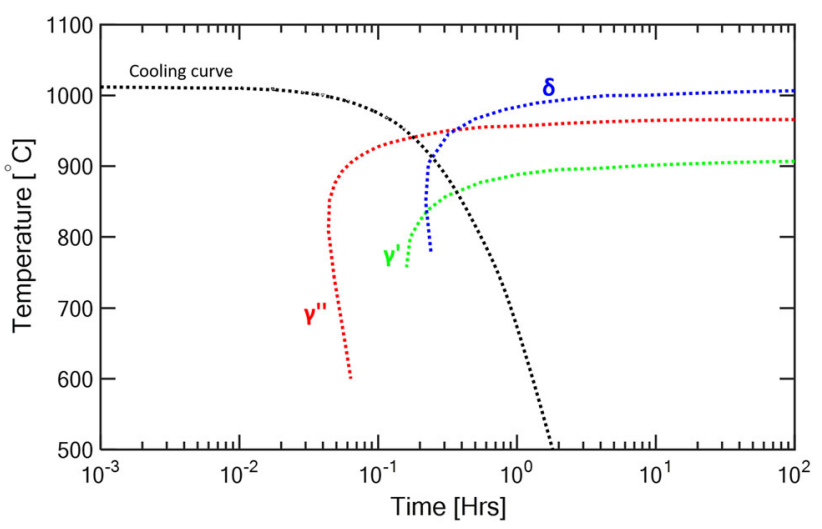

Fig. 10 - CCT diagram created using JMatPro that relates to the homogenized part of the microstructure. The dotted line represents the 0.5 pct transformation related to the nominal composition of the Alloy. The cooling curve has been created from the thermocouple measurement in the cooling stage in Fig. 1.

more homogeneous and reached the nominal values. Therefore, the CCT curves generated based on the nominal composition can be used to describe the homogenized part of the microstructure of the sample (below $1800 \mu \mathrm{m}$ from the top of the surface). According to Figure 10 , the $\gamma^{\prime} / \gamma^{\prime \prime}$ particles precipitated earlier and increased in size more rapidly in the homogenized part of the sample compared with the dendrite core of the "solidified" microstructure. Therefore, the hardness was higher in the homogenized part of the microstructure compared with the dendrite core of the "solidified" microstructure. This prediction was confirmed by previously reported hardness observations. ${ }^{[19]}$

According to the CCT curves obtained for $\gamma^{\prime}$ and $\gamma^{\prime}$, as shown in Figure 9, $\gamma^{\prime}$ started to precipitate earlier than $\gamma^{\prime \prime}$. However, the CCT curves obtained for the nominal composition of the alloy (see Figure 10) showed that the precipitation of $\gamma^{\prime \prime}$ occurred earlier than that of $\gamma^{\prime}$. A similar accelerated precipitation of $\gamma^{\prime}$ before that of $\gamma^{\prime \prime}$ was reported previously ${ }^{[29]}$ for $\mathrm{Ni}-\mathrm{Cr}-\mathrm{Fe}$ alloys with compositions approximating that of Alloy 718. This phenomenon is linked to high $\mathrm{Ti}+\mathrm{Al} / \mathrm{Nb}$ ratios ${ }^{[29]}$ and in the present study, this ratio was around 1.03 and 2.35 for the nominal and interdendritic compositions, respectively, which could have accelerated the precipitation of $\gamma^{\prime}$ before that of $\gamma^{\prime \prime}$ in the interdendritic region. However, no experimental research has been performed to confirm the results obtained in the present study.

\section{CONCLUSION}

In this study, we investigated the microstructural evolution during EBM of Alloy 718 by microstructure modeling. Multiphase-field modeling and precipitation kinetics modeling using JMatPro were also conducted. We provided the following conclusions based on the results.

The as-built microstructure of the EBM Alloy 718 exhibited a microstructure gradient from the top to the bottom of the sample.
- The high bed temperature during production resulted in an "in situ" heat treatment, which had a homogenization effect on the solidified microstructure.

- Due to the smaller PDAS and relatively low Laves phase size, EBM Alloy 718 exhibited more rapid homogenization compared with the cast or wrought material, which may facilitate the design of specific heat treatment protocols for EBM printed Alloy 718.

- The segregation of the alloying elements into the interdendritic region (close to the Laves phase) changed the precipitation kinetics of the alloy and led to the formation of high amounts of $\gamma^{\prime} / \gamma^{\prime \prime}$ and $\delta$ in this region compared with the dendritic core.

- This combined approach based on multiphase-field modeling using MICRESS and transformation kinetic modeling using JMatPro is a viable method for obtaining insights into microstructural formation during the additive manufacturing of nickel-based superalloys and subsequent heat treatments.

\section{ACKNOWLEDGMENTS}

The authors would like to thank Dr. Bernd Böttger and Dr. Eiken, Janin at Access e.V., Aachen, Germany for valuable discussions and inputs regarding the modeling work using MICRESS. Funding from the European Regional Development Fund for project 3Dprint and from the KK Foundation (Stiftelsen för Kunskaps-och Kompetensutveckling) for project SUMAN-Next is also acknowledged.

\section{OPEN ACCESS}

This article is distributed under the terms of the Creative Commons Attribution 4.0 International License (http://creativecommons.org/licenses/by/4.0/), which permits unrestricted use, distribution, and reproduction in any medium, provided you give appropriate credit to the original author(s) and the source, provide a link to the Creative Commons license, and indicate if changes were made.

\section{REFERENCES}

1. W.J. Sames, F.A. List, S. Pannala, R.R. Dehoff, and S.S. Babu: Int. Mater. Rev., 2016, vol. 6608, pp. 1-46.

2. M.M. Attallah, R. Jennings, X. Wang, and L.N. Carter: $M R S$ Bull., 2016, vol. 41, pp. 758-64.

3. C. Körner: Int. Mater. Rev., 2016, vol. 61, pp. 361-77.

4. G.A. Knorovsky, M.J. Cieslak, T.J. Headley, A.D. Romig, and W.F. Hammetter: Metall. Trans. A, 1989, vol. 20, pp. 2149-58.

5. W.J. Sames, K.A. Unocic, R.R. Dehoff, T. Lolla, and S.S. Babu: J. Mater. Res., 2014, vol. 29, pp. 1920-30.

6. M.M. Kirka, K.A. Unocic, N. Raghavan, F. Medina, R.R. Dehoff, and S.S. Babu: JOM, 2016, vol. 68, pp. 1012-20.

7. I. Steinbach: Model. Simul. Mater. Sci. Eng., 2009, vol. 17, pp. 73001-31. 
8. J. Kundin, L. Mushongera, and H. Emmerich: Acta Mater., 2015, vol. 95 , pp. $343-56$.

9. R. Acharya, J.A. Sharon, and A. Staroselsky: Acta Mater., 2017, vol. 124, pp. 360-71.

10. J. Eiken, B. Böttger, and I. Steinbach: Phys. Rev. E, 2006, vol. 73, p. 066122

11. J. Eiken: Shaker Verlag GmbH, Germany, 2010.

12. B. Böttger, J. Eiken, and M. Apel: Comput. Mater. Sci., 2015, vol. 108, pp. 283-92.

13. Thermo-Calc Software, http://www.thermocalc.com/. Accessed 25 May 2018.

14. N. Warnken, D. Ma, A. Drevermann, R.C. Reed, S.G. Fries, and I. Steinbach: Acta Mater., 2009, vol. 57, pp. 5862-75.

15. Micress Group: MICRESS 6.4 - User Guide Volume II: Running MICRES, vol. 2.

16. J.K. Tien and T. Caulfield: Superalloys, Supercomposites and Superceramics, Academic Press, New York, 1989.

17. SAE: AMS5383E-Nickel Alloy, Corrosion and Heat-Resistant, Investment Castings, 2012

18. JMatPro, https://www.sentesoftware.co.uk/jmatpro. Accessed 21 Octob 2018.

19. D. Deng, R.L. Peng, H. Söderberg, and J. Moverare: Mater. Des., 2018, vol. 160, pp. 251-61.
20. N. Raghavan, R. Dehoff, S. Pannala, S. Simunovic, M. Kirka, J. Turner, N. Carlson, and S.S. Babu: Acta Mater., 2016, vol. 112, pp. 303-14.

21. T. Antonsson and H. Fredriksson: Metall. Mater. Trans. B, 2005, vol. 36 , pp. 85-96.

22. J.F. Radavich: Superalloys 718 Metallurgy and Applications, TMS, Pittsburgh, 1989, pp. 229-40.

23. P. Nie, O.A. Ojo, and Z. Li: Acta Mater., 2014, vol. 77, pp. 85-95.

24. Y.S. Lee and W. Zhang: Addit. Manuf., https://doi.org/10.1016/ j.addma.2016.05.003.

25. G. Asala, A.K. Khan, J. Andersson, and O.A. Ojo: Metall. Mater. Trans. A, 2017, vol. 48, pp. 4211-28.

26. R.G. Carlson and J.F. Radavich: Superalloys 718 Metall. Appl., 1989, pp. 79-95.

27. V. Beaubois, J. Huez, S. Coste, O. Brucelle, and J. Lacaze: Mater. Sci. Technol., 2004, vol. 20, pp. 1019-26.

28. Y. Tian, D. McAllister, H. Colijn, M. Mills, D. Farson, M. Nordin, and S. Babu: Metall. Mater. Trans. A, 2014, vol. 45, pp. $4470-83$.

29. R. Cozar and A.A. Pineau: Metall. Trans., 1973, vol. 4, pp. 47-59.

Publisher's Note Springer Nature remains neutral with regard to jurisdictional claims in published maps and institutional affiliations. 